\title{
Bending Motions in the Dihalides of Group II Metals
}

\author{
R. STEPHEN BERRY \\ Department of Chemistry, University of Michigan, Ann Arbor, Michigan
}

(Received August 11, 1958)

\begin{abstract}
A polarizable ion model is used to calculate the force constants for bending in the dihalides of the secondgroup metals. The results indicate that the bending constant is a sensitive function of the polarizability of the metal. The calculated constants are in moderate agreement with the experimental values. Some of the implicit assumptions of the model are discussed together with the implications of the calculated results.
\end{abstract}

\section{INTRODUCTION}

$\mathbf{T}$ THE problem of calculating nuclear vibration frequencies from electronic structures is an old and troublesome one. The real difficulty in such a calculation is the fact that the nuclei move in an effective potential field, a potential field which changes with every new relative position of the nuclei. Consequently accurate vibration frequencies can be derived only after the electronic Schroedinger equation is solved for many nuclear configurations in the region of the potential minimum. This task is occasionally carried out for diatomic molecules, but, excepting the water molecule, has not been done for polyatomic molecules. In a situation like this, it is of ten worthwhile to examine a simple model consistent with our general notion of a system. Specifically, we shall use a polarizable-ion model to treat the bending motions in the series of Group II dihalides. We hope to indicate that such a model does provide a reasonable first approximation for calculation of the bending force constants, and show what terms will be important in a full quantummechanical treatment of this problem. And, of course, we wish to demonstrate the point that prompted this work initially: that the high values of the bending force constants of $\mathrm{MgCl}_{2}, \mathrm{BeCl}_{2}$, and $\mathrm{BeF}_{2}$ found by Büchler and Klemperer ${ }^{1}$ are entirely consistent with the much lower values found for $\mathrm{HgCl}_{2}, \mathrm{HgBr}_{2}$, and $\mathrm{HgI}_{2}{ }^{2-4}$

In the following sections we shall outline the model and the calculation, and present the tabulated results. In the final section, we discuss the implications of the results, the shortcomings of the model, and its potential for further exploitation.

\section{MODEL}

The model for this calculation is quite similar to that used by Hund in his treatment of triatomic molecules, ${ }^{5}$ and discussed by Debye with reference to the structure of water. ${ }^{6}$ We assume that our triatomic molecules

1 A. Büchler and W. Klemperer, J. Chem. Phys. 29, 121 (1958).

2 M. Wehrli, Helv. Phys. Acta 11, 339 (1938).

3 M. Wehrli, Helv. Phys. Acta 13, 153 (1940).

4 H. Sponer and E. Teller, J. Chem. Phys. 7, 382 (1939).

${ }^{6}$ F. Hund, Z. Physik 31, 81 (1925).

${ }^{B}$ P. Debye, Polar Molecules (The Chemical Catalogue Company, New York, 1929). may be represented as three hard spheres in contact, and attribute to each a charge a dipole polarizability and a quadrupole polarizability. The magnesium chloride molecule, for example, is represented by two negative, polarized chloride ions flanking a doubly positive, polarized magnesium ion. The equilibrium configuration is of course linear.

To evaluate the force constant for the bending motion, we displace one halide ion away from its equilibrium position. The displacement is small but finite, and follows the circular arc corresponding to a constant halide-metal bond length. Such a motion runs along the normal bending coordinate in valence force field coordinates. ${ }^{7}$ The net restoring force in the direction tangential to the circular arc is proportional to the displacement $\Delta$ (and, for the time being, we neglect terms in $\Delta^{2}$ ). The constant of proportionality so defined is simply half of the usual bending constant $k_{\delta} / l^{2}$.

Figure 1 is a sketch of the model, with halide $\mathrm{X}_{1}^{-}$ displaced downward from its equilibrium position in line with metal $\mathrm{M}^{++}$and halide $\mathrm{X}_{2}^{-}$.

\section{CALCULATIONS}

The restoring force in this model depends rigorously on all the multipole interactions between pairs of ions. We shall include charge-charge, charge-dipole, chargequadrupole, dipole-dipole, dipole-quadrupole, and quadrupole-quadrupole interactions, and briefly discuss the terms we neglect.

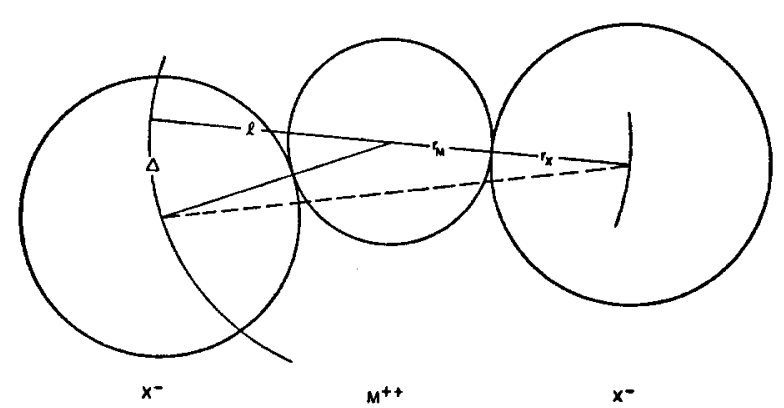

FIG. 1. The model of an ionic $\mathrm{MX}_{2}$ molecule, displaced from linear equilibrium position.

${ }^{7}$ G. Herzberg, Infrared and Raman Spectra (D. Van Nostrand Company, Inc., Princeton, New Jersey, 1945). 
There is only one charge-charge interaction, namely that due to the slight decrease in halide-halide distance when bending occurs. Four charge-induced dipole interactions contribute to the bending force:

(a) one halide interacting with the dipole induced by the metal in the other halide,

(b) one halide interacting with the dipole it induces in the other halide,

(c) a halide interacting with the dipole induced by the other halide in the metal, and

(d) the metal interacting with a dipole induced in one halide by the other.

The charge-quadrupole interactions which contribute to the bending force are only three in number:

(a) one halide interacting with the quadrupole it induces in the other halide,

(b) a halide interacting with the quadrupole induced by the other halide in the metal, and

(c) the metal interacting with the quadrupole moment induced in one halide by the other.

Six dipole-dipole interactions contribute to the bending force, of which three are halide-halide and three, metalhalide interactions. These, together with the chargecharge, charge-dipole, and charge-quadrupole interactions, are shown schematically in Fig. 2. The tangential arrows indicate the direction in which the force tends to move the nucleus in question.

The force constant for bending may be represented as a series in inverse powers of the halide-metal bond length, $l$, as

$$
k_{\delta} / l^{2}=k^{(0)}+k^{(1)}+k^{(2)}+k^{(3)}+k^{(3) \prime}+\cdots .
$$

The terms correspond to the following interactions, respectively: $k^{(0)}$, to charge-charge interactions; $k^{(1)}$, to interaction of charges with dipoles induced by monopoles; $k^{(2)}$, to interaction of charges with quadrupoles induced by monopoles; $k^{(3)}$, to the first induced dipoleinduced dipole interaction, and $k^{(3) '}$, to the interaction of charges with dipoles induced by induced dipoles. The last named, $k^{(3) \prime}$, can be thought of as a correction to $k^{(1)}$, due to the fact that induced dipoles arise from fields of polarized, not spherical, ions.

These interactions have the forms

$$
\begin{gathered}
k^{(0)}=e^{2} / 4 l^{3}, \\
k^{(1)}=\left(e^{2} / 2 l^{6}\right)\left[\alpha_{d}(x)-8 \alpha_{d}(m)\right], \\
k^{(2)}=\left(6 e^{2} / l^{3}\right)\left[\frac{31}{256} \alpha_{q}(x)-\alpha_{q}(m)\right], \\
k^{(3)}=\left(e^{2} \alpha_{d}(x) / l^{9}\right)\left[\frac{18}{32} \alpha_{d}(x)-\frac{27}{2} \alpha_{d}(m)\right],
\end{gathered}
$$

and

$$
k^{(3) \prime}=\left(-7 e^{2} \alpha_{d}(x) / l^{9}\right)\left[\frac{\mathrm{g}}{64} \alpha_{d}(x)+\alpha_{d}(m)\right] .
$$

Here, $e$ is the electronic charge, $l$ is the halide-metal bond length, $\alpha_{d}(x)$ and $\alpha_{d}(m)$ the dipole polarizabilities of the halide and metal, respectively, and $\alpha_{q}(x)$ and
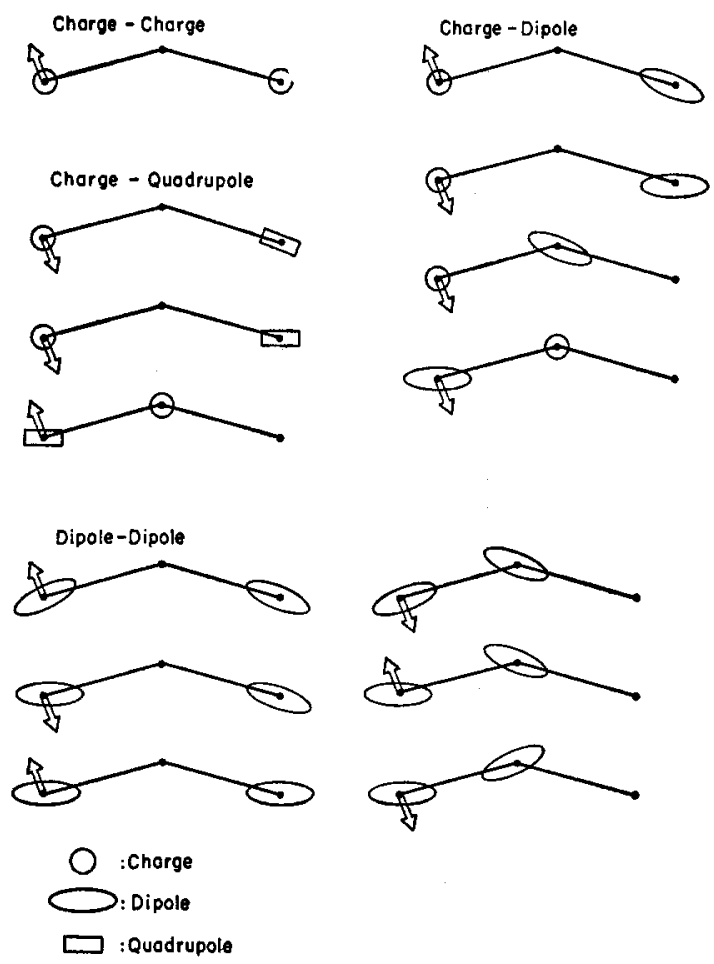

Fig. 2. Schematic representation of the lower order multipole interactions.

$\alpha_{2}(m)$ the halide and metal quadrupole polarizabilities as defined by Sternheimer. ${ }^{8}$

In evaluating the expressions (2)-(6) and (7) and (8) following, we used the results of Akishin and Spiridonov ${ }^{9}$ for bond lengths derived from electron diffraction. The metal-dipole polarizabilities were derived from the refractivities given by Van Vleck, ${ }^{10}$ and for the heaviest metals, lower values were considered also, to allow for saturation. The corresponding halide values were taken from the results of Rice and Klemperer. ${ }^{11}$ These were chosen in preference to the crystal values since they include effects of saturation which must occur in the strong fields of close neighboring ions. For quadrupole polarizabilities, the values calculated by Sternheimer ${ }^{8,12}$ were used whenever possible, and estimates were made for those remaining. Unfortunately, there seemed to be no meaningful way to introduce saturation into the quadrupole polarizabilities, so the values used will simply set upper limits to the quadrupole contributions to the force constants. The chosen values of the polarizabilities are given in Table I.

${ }^{8}$ R. M. Sternheimer, Phys. Rev. 96, 951 (1954).

9 P. A. Akishin and V. P. Spiridonov, Kristallografiya 2, 475 (1957); also Chem. Abstracts 51, 17297i (1957).

$10 \mathrm{~J}$. H. Van Vleck, The Theory of Electric and Magnetic Susceptibilities (Oxford University Press, London, 1932).

${ }^{11} \mathrm{~S}$. A. Rice and W. Klemperer, J. Chem. Phys. 27, 573 (1957). 12 R. M. Sternheimer, Phys. Rev. 107, 1565 (1957). 
TABLE I. Ionic polarizabilities.

\begin{tabular}{|c|c|c|}
\hline Ion & $\alpha_{d}\left(A^{3}\right)$ & $\alpha_{q}\left(A^{b}\right)$ \\
\hline $\begin{array}{l}\mathrm{Be}^{++} \\
\mathrm{Mg}^{++} \\
\mathrm{Ca}^{++} \\
\mathrm{Sr}^{++} \\
\mathrm{Ba}^{++} \\
\mathrm{Zn}^{++} \\
\mathrm{Cd}^{++} \\
\mathrm{Hg}^{++}\end{array}$ & $\begin{array}{l}7.9 \times 10^{-3 \mathrm{a}} \\
9.43 \times 10^{-2 \mathrm{a}} \\
0.5^{\mathrm{o}} \\
0.86^{\mathrm{a}} \\
1.56^{\mathrm{a}} \\
0.285^{\mathrm{a}} \\
1.09^{\mathrm{a}} \\
1.244^{\mathrm{a}} \\
1,0.5^{\mathrm{d}}\end{array}$ & $\begin{array}{l}6.4 \times 10^{-4 b} \\
7 \times 10^{-3 b} \\
0.02^{\mathrm{d}} \\
0.06^{\mathrm{d}} \\
0.10^{\mathrm{d}} \\
0.05^{\mathrm{d}} \\
0.10^{\mathrm{d}} \\
0.5^{\mathrm{d}}\end{array}$ \\
\hline $\begin{array}{l}\mathrm{F}^{-} \\
\mathrm{Cl}^{-} \\
\mathrm{Br}^{-} \\
\mathrm{I}^{-}\end{array}$ & $\begin{array}{l}1.0^{\mathrm{a}} \\
2.8^{\mathrm{o}} \\
3.7^{\circ} \\
5.7^{\circ}\end{array}$ & $\begin{array}{l}1.0^{\mathrm{d}} \\
13.77^{\mathrm{b}} \\
30^{\mathrm{d}} \\
50^{\mathrm{d}}\end{array}$ \\
\hline
\end{tabular}

- See reference 10 .

b See references 8 and 12 .

- Estimated; reference 10 gives 0.47 , but reference 8 quotes higher values.

a Estimated.

- See reference 11.

In addition to the terms discussed heretofore, the dipole-quadrupole contribution $k^{(5)}$, and the quadrupole-quadrupole contribution $k^{(7)}$, to the bending force constant, have been calculated. For these we arrive at the expressions

$$
\begin{aligned}
k^{(5)}=\left(e^{2} / l^{11}\right)\left[\frac{105}{156} \alpha_{d}(x) \alpha_{q}(x)+\right. & \frac{231}{8} \alpha_{d}(x) \alpha_{q}(m) \\
& \left.+\frac{177}{16} \alpha_{d}(m) \alpha_{q}(x)\right]
\end{aligned}
$$

and

$k^{(7)}=\left(e^{2} / l^{13}\right) \alpha_{q}(x)\left[\frac{5943}{16384} \alpha_{q}(x)-\frac{2595}{32} \alpha_{q}(m)\right]$.

These have been included in the force constant, but it is important to note that other terms of comparable orders in $l$ have been omitted. Specifically, the chargeoctopole term, depending on $l^{-10}$, and the dipoleoctopole term, depending on $l^{-12}$, would contribute $k^{(4)}$ and $k^{(6)}$ to $k_{\delta} / l^{2}$. These two were omitted because of the dearth of information regarding the octopole polarizabilities of ions. Other terms omitted include the higher analogs of $k^{(3) '}$; the first of these is the correction to the dipole-dipole term due to the fact that the fields inducing the interacting dipoles are due to ions with induced dipoles. This term depends on $l^{-12}$. The correction to the charge-quadrupole interaction analogous to $k^{(3) \prime}$ falls at $l^{-13}$. We have also omitted explicit mention of the saturation terms; these are discussed in the next section.

Table II contains values for the individual contributions for representative molecules, to show how the series converges and how different values of polarizabilities of the heavy metals affect the force constant. In Table III are the bond lengths and force constants; some allowance was made for saturation of metal polarizabilities, as noted in the table references.

\section{DISCUSSION}

The first point one might note is the way in which the various terms (3)-(8) depend on the polarizabilities of the halide and metal ions. Because of the proximity of metal to halide, in any expression for a $k^{(j)}$, the coefficient of the metal polarizability is invariably much larger than that multiplying the halide polarizability. And in addition, the sign of the term containing $\alpha(m)$ is generally negative, while that of the halide term is positive. Thus, the more polarizable the halide, the stiffer the molecule, and the more polarizable the metal, the more easily the molecule bends. We note from Table I that the metal polarizabilities are generally much smaller than those of the halides. Consequently the halide contribution tends to dominate, except for the large, highly polarizable metals. As a result of this strong dependence of $k_{\delta} / l^{2}$ on $\alpha_{d}(m)$ and $\alpha_{q}(m)$, we note in Table II that the calculated and observed constants do drop very sharply as the metal size and polarizability increase.

The inability to account for saturation of quadrupole polarizability makes itself felt in those cases where bond lengths are small and $\alpha_{q}(x)$ 's are large, namely in the case of $\mathrm{BeCl}_{2}, \mathrm{BeBr}_{2}$, and $\mathrm{BeI}_{2}$. The high calculated values of the bending constant can be attributed primarily to this effect. Again, in the case of the calculated negative values of $k_{\delta} / l^{2}$, the overestimation of $\alpha_{q}(m)$ makes the molecule seem too soft. This is particularly evident in the case of the cadmium salt, where the bond length is quite short and the crystal polarizability is extremely large.

It is interesting to note that the quadrupole terms $k^{(2)}$ and $k^{(5)}$ are quite large, often larger than the dipole terms $k^{(1)}$ and $k^{(3)}$. In general, the quadrupole-quadrupole interaction is considerably smaller than the lower order terms.

The best results are obtained for those molecules in which interionic penetration is relatively small, and the bond length is relatively large. The first of these is important because the expansion of the interaction of charge clouds is only truly valid for nonoverlapping distributions. The second is important so that saturation is minimized, and so that the expansion in inverse powers of the bond length converges as rapidly as possible. These conditions are probably optimized for the magnesium salts, and perhaps for the calcium salts.

With regard to saturation, one may express the polarizability of any given order in a power series in the appropriate derivative of the field. Thus one can write the dipole polarizability as a Maclaurin series in the field itself, the quadrupole polarizability terms as a series in powers of the field gradient, and so on. For our purposes, the fields are those of neighboring ions, so they and their derivatives are expressible in inverse powers of $l$. Thus the saturation terms could be included directly and explicitly in the expansion of $k_{\delta} / l^{2}$. In this expansion, we find that the first dipole satura- 
TABLE II. Terms in the calculation of $k_{\delta} / l^{2}$.

\begin{tabular}{|c|c|c|c|c|c|c|c|}
\hline Molecule & $k^{(0)}+k^{(1)}$ & $k^{(2)}$ & $k^{(a)}$ & $k^{(3)^{\prime}}$ & $k^{(5)}$ & $k^{(7)}$ & $k \delta / l^{2}$ \\
\hline $\mathrm{BeF}_{2}$ & 0.354 & 0.113 & 0.055 & -0.117 & 0.044 & 0.009 & 0.460 \\
\hline $\mathrm{BeCl}_{2}$ & 0.217 & 0.262 & 0.065 & -0.118 & 0.133 & 0.108 & 0.668 \\
\hline $\mathrm{MgF}_{2}$ & 0.113 & 0.016 & -0.004 & -0.022 & 0.008 & $-0.000_{3}$ & 0.112 \\
\hline $\mathrm{MgCl}_{2}$ & 0.078 & 0.045 & 0.002 & -0.020 & 0.018 & 0.006 & 0.129 \\
\hline $\mathrm{MgBr}_{2}$ & 0.066 & 0.055 & 0.004 & -0.017 & 0.021 & 0.010 & 0.127 \\
\hline $\mathrm{CaF}_{2}$ & 0.021 & 0.004 & -0.018 & -0.013 & 0.004 & $-0.000_{3}$ & $(-0.001)$ \\
\hline $\mathrm{CaCl}_{2}$ & 0.031 & 0.014 & -0.008 & -0.010 & 0.010 & 0.001 & 0.037 \\
\hline $\mathrm{BaCl}_{2}$ & -0.039 & $0.000_{3}$ & -0.024 & -0.008 & 0.006 & -0.000 & $(-0.004)$ \\
\hline $\mathrm{BaI}_{2}$ & 0.010 & 0.008 & -0.007 & -0.006 & 0.007 & $0.000_{3}$ & 0.013 \\
\hline $\mathrm{CdF}_{2}$ & -0.077 & 0.001 & -0.073 & -0.044 & 0.021 & -0.003 & $(-0.175)$ \\
\hline $\mathrm{HgF}_{2}^{\mathrm{B}}$ & 0.012 & -0.028 & -0.040 & -0.014 & 0.015 & -0.006 & $(-0.019)$ \\
\hline $\mathrm{HgCl}_{2}{ }^{\mathrm{a}}$ & 0.038 & 0.021 & -0.019 & -0.023 & 0.036 & -0.024 & 0.030 \\
\hline $\mathrm{HgF}_{2}^{\mathrm{b}}$ & -0.036 & -0.015 & -0.041 & -0.025 & 0.019 & -0.006 & $(-0.104)$ \\
\hline $\mathrm{HgCl}_{2} \mathrm{~b}$ & 0.006 & 0.021 & -0.044 & -0.036 & 0.055 & -0.024 & $(-0.021)$ \\
\hline
\end{tabular}

s Based on $\alpha_{d}\left(\mathrm{Hg}^{++}\right)=0.5$.

b Based on $\alpha_{d}\left(\mathrm{Hg}^{++}\right)=1.0$.

tion term lies two powers of $l$ further out than the constant term, the first quadrupole term is three powers of $l$ further out than the constant term, and so on. Therefore if saturation is included explicitly, our series of $k^{(j)}$ 's would contain a term $k_{1}^{(1)}$ proportional to $l^{-8}$; $k_{2}{ }^{(1)}, k_{1}{ }^{(2)}$, and $k_{1}{ }^{\left({ }^{3)}\right.}$ proportional to $l^{-11}, k_{1}{ }^{(5)}$ and $k_{2}{ }^{(3)}$ proportional to $l^{-13}$ and higher terms. The first of these are implicitly included in the present calculation because of our choice of halide polarizabilities.

The salts of Group II metals are often discussed in terms of their degree of covalency. ${ }^{13}$ In such a treatment, the term "ionic character" refers to the fraction of the exact or best approximate wave function which corresponds to spherical, unpolarized ions in their ground states. The functions which usually form the basis for an approximate molecular function include the ground-state ion functions and a few neutral atom functions, enough to give the desired hybridizations of the atoms. The complete set of such functions is of course the set of exact solutions for the molecule at extremely large separations of the nuclei.

The polarized-ion model is based implicitly on a different set of functions, namely the set of products of ground- and excited-state functions of isolated ions in free space. These functions would be obtained by solving the Schroedinger equation for each ion in the molecule, in the absence of all the others. In the ionicplus-covalent model, the number of electrons associated with a given nucleus may change, depending on the state of the system; in the polarized-ion model, each nucleus is always the center for the same number of electrons.

The higher states need not be displayed explicitly in the polarized-ion model. Sternheimer's calculations of polarized-ion wave functions, ${ }^{8,12}$ being solutions of the perturbation equation, need not be solved in terms of

${ }^{13}$ See, for example, Gowenlock, Polanyi, and Warhurst, Proc. Roy. Soc. (London) A219, 270 (1953). any particular set of unperturbed, stationary state wave functions.

It is important to realize how the basis functions implicit in the polarized ion model differ from those for

TABLE III. Bond lengths and force constants. Except where noted, bond lengths are taken from reference 9. Saturation of metal polarization is included only in case of mercury.

\begin{tabular}{|c|c|c|c|}
\hline Molecule & $l$ & $k_{\delta} / l^{2}($ calc $)$ & $k_{\delta} / l^{2}(\exp )$ \\
\hline $\begin{array}{l}\mathrm{BeF}_{2} \\
\mathrm{BeCl}_{2} \\
\mathrm{BeBr}_{2} \\
\mathrm{BeI}_{2} \\
\mathrm{MgF}_{2} \\
\mathrm{MgCl}_{2} \\
\mathrm{MgBr}_{2} \\
\mathrm{MgI}_{2} \\
\mathrm{CaF}_{2} \\
\mathrm{CaCl}_{2} \\
\mathrm{CaBr}_{2} \\
\mathrm{CaI}_{2} \\
\mathrm{SrF}_{2} \\
\mathrm{SrCl}_{2} \\
\mathrm{SrBr}_{2} \\
\mathrm{SrI}_{2} \\
\mathrm{BaF}_{2} \\
\mathrm{BaCl}_{2} \\
\mathrm{BaBr}_{2} \\
\mathrm{BaI}_{2} \\
\mathrm{ZnF}_{2} \\
\mathrm{ZnCl}_{2} \\
\mathrm{ZnBr}_{2} \\
\mathrm{ZnI}_{2} \\
\mathrm{CdF}_{2} \\
\mathrm{CdCl}_{2} \\
\mathrm{CdBr}_{2} \\
\mathrm{CdI}_{2} \\
\mathrm{HgF}_{2} \\
\mathrm{HgCl}_{2} \\
\mathrm{HgBr}_{2} \\
\mathrm{HgI}_{2}\end{array}$ & $\begin{array}{l}1.40 \mathrm{~A} \\
1.75 \\
1.91 \\
2.18 \\
1.77 \\
2.18 \\
2.34 \\
2.55 \\
2.10 \\
2.51 \\
2.67 \\
2.88 \\
2.20 \\
2.67 \\
2.82 \\
3.03 \\
2.32 \\
2.82 \\
2.99 \\
3.20 \\
1.81 \\
2.05 \\
2.21 \\
2.38 \\
1.97 \\
2.21 \\
2.37 \\
2.55 \\
2.08 \\
2.29 \\
2.41 \\
2.59\end{array}$ & $\begin{array}{c}0.46 \mathrm{md} / \mathrm{A} \\
0.67 \\
0.72 \\
0.42 \\
0.11 \\
0.13 \\
0.13 \\
0.12 \\
(-0.001) \\
0.04 \\
0.05 \\
0.05 \\
(-0.03) \\
0.02 \\
0.03 \\
0.03 \\
(-0.07) \\
-0.004) \\
0.007 \\
0.013 \\
0.006 \\
0.13 \\
0.17 \\
0.17 \\
(-0.17) \\
(-0.02) \\
0.05 \\
0.07 \\
(-0.02)^{\mathrm{b}} \\
0.03 \mathrm{~b} \\
0.05^{\mathrm{c}} \\
0.05 \mathrm{c}\end{array}$ & $\begin{array}{l}0.038 \\
0.022 \\
0.018\end{array}$ \\
\hline
\end{tabular}

- Estimated.

b Based on $\alpha_{d}\left(\mathrm{Hg}^{++}\right)=0.5$.

- Based on $\alpha_{d}\left(\mathrm{Hg}^{++}\right)=1.0$. 
the three separated nuclei. In the set based on separated nuclei, the various atomic states are included. These states cannot appear in the ionic model since each member of the basis is a product of factors each of which is a solution to the Schroedinger equation of single free ion. The ionic model set consists therefore of products of ground-state or excited (presumably continuum) free negative ion functions with any and all of the positive ion functions. The ionic set and the ionic-plus-covalent set do have certain functions in common, namely the products of ground-state negative ion functions with all the bound positive-ion functions. All the other functions must be different.

Since the two descriptions are based on different complete sets, the basis functions of one description will not be orthogonal to those of the other. This means especially that inclusion of covalent character must do at least part of the same job as inclusion of polarization. As long as only a few terms are included in either treatment, we shall expect their best approximate wave functions to be linearly independent, but quite nonorthogonal. Until one knows their degree of nonorthogonality, it is impossible to tell just how much is omitted by any approximate polarized ion function that is included in a covalent function. Moreover either set, being complete, must ultimately provide a correct molecular wave function, so that in any given problem, the choice between the two is one of convenience and of rapidity of convergence. The latter can only be determined by successive application of the models to more and more sensitive phenomena.

We might mention at this point that the ionic model does contain one major inconsistency as it is applied in the present work. The multipole interactions are treated as though the charge distributions of the ions do not overlap. In fact, interpenetration is quite important, especially for the larger ions. This penetration is implicitly but only partially taken into account in the values of polarizabilities. But this is not at all a satisfactory thing to do, and a critical examination of the meaning of polarization of overlapping charge distributions would be in order, perhaps in terms of an expansion like that of Buehler and Hirschfelder. ${ }^{14}$

Finally, we return to an examination of Table III, to look at the fluorides of the heavier metals. We see that $k_{\delta} / l^{2}$ is negative for some of these, implying that the molecules should be bent. This is contrary to the experimental evidence of Akishin and Spiridonov, ${ }^{9}$ who report on all the Group II dihalides except $\mathrm{MgI}_{2}$ and $\mathrm{HgF}_{2}$, and find them to be linear. We attribute this disparity mainly to two factors, our inability to evaluate dipole saturation effects in the heavy metals, and to the neglect of octopole terms $k^{(4)}$ and $k^{(6)}$. It is interesting to note, however, that $\mathrm{HgF}_{2}$ is one of the most extreme cases, in terms of large metal and small halide polarizabilities. The prediction of a bent structure is probably an artifact of the model, but might well be checked experimentally.

\section{ACKNOWLEDGMENTS}

The author would like to express his appreciation to Dr. W. Klemperer and Mr. A. Büchler for many stimulating discussions, for making available a preprint of their work, and to Mr. Büchler, for making the author aware of the paper of Akishin and Spiridonov.

${ }^{14}$ R. J. Buehler and J. O. Hirschfelder, Phys. Rev. 83, 628 (1951); ibid. 85, 149 (1952). 